\title{
Maternal cardioautonomic responses during and following exercise throughout pregnancy
}

\begin{tabular}{|c|c|}
\hline Journal: & Applied Physiology, Nutrition, and Metabolism \\
\hline Manuscript ID & apnm-2018-0397.R1 \\
\hline Manuscript Type: & Article \\
\hline $\begin{array}{r}\text { Date Submitted by the } \\
\text { Author: }\end{array}$ & 03-Aug-2018 \\
\hline Complete List of Authors: & $\begin{array}{l}\text { Purdy, Graeme; University of Alberta, Faculty of Kinesiology, Sport, and } \\
\text { Recreation } \\
\text { James, Marina; University of Alberta } \\
\text { Wakefield, Paige; University of Alberta } \\
\text { Skow, Rachel; University of Alberta, Faculty of Physical Education and } \\
\text { Recreation } \\
\text { Van Diepen, Sean; University of Alberta Faculty of Medicine and } \\
\text { Dentistry } \\
\text { May, Linda; East Carolina University } \\
\text { Davenport, Margie; University of Alberta, Faculty of Physical Education } \\
\text { and Recreation; University of Alberta, Program for Pregnancy and } \\
\text { Postpartum Health } \\
\text { Steinback, Craig; University of Alberta, Faculty of Physical Education and } \\
\text { Recreation }\end{array}$ \\
\hline Keyword: & $\begin{array}{l}\text { arterial baroreflex, heart rate variability, exercise recovery, autonomic } \\
\text { nervous system < nervous system, pregnancy }\end{array}$ \\
\hline $\begin{array}{r}\text { Is the invited manuscript for } \\
\text { consideration in a Special } \\
\text { Issue? : }\end{array}$ & Not applicable (regular submission) \\
\hline
\end{tabular}

\section{SCHOLARONE Manuscripts}




\section{Maternal cardioautonomic responses during and following exercise throughout pregnancy}

Graeme M Purdy ${ }^{1,2}$, Marina A James ${ }^{1,2}$, Paige K Wakefield ${ }^{1-3}$, Rachel J Skow ${ }^{1-3}$, Sean Van Diepen $^{4}$, Linda E May ${ }^{5}$, Margie H Davenport ${ }^{1-3}$ and Craig D Steinback ${ }^{1-3}$.

${ }^{1}$ Neurovascular Health Lab, Program for Pregnancy and Postpartum Health, Faculty of Kinesiology, Sport, and Recreation, University of Alberta, Edmonton, AB, T6G 2H9, Canada.

${ }^{2}$ Women and Children's Health Research Institute, University of Alberta, Edmonton, AB, T6G 1C9, Canada.

${ }^{3}$ Alberta Diabetes Institute, University of Alberta, Edmonton, AB, T6G 2E1, Canada.

${ }^{4}$ Faculty of Medicine and Dentistry, Department of Critical Care and Division of Cardiology, University of Alberta, Edmonton, AB, T6G 2R7, Canada.

${ }^{5}$ Division of Foundational Sciences and Research, East Carolina University, Greenville, NC, 27858, USA.

\section{Corresponding Author}

Craig D. Steinback, PhD

Assistant Professor

Program for Pregnancy and Postpartum Health

Faculty of Kinesiology, Sport, and Recreation, University of Alberta

1-059D Li Ka Shing Centre for Health Research Innovation

Edmonton, Alberta, Canada

T6G 2E1

Tel: (780) 492-5553

Email: craig.steinback@ualberta.ca 


\section{Abstract}

Blood pressure regulation during pregnancy is poorly understood. Cardiovagal baroreflex gain (BRG) is an important contributor to blood pressure regulation via its influence on heart-rate. Heart-rate fluctuations occur in response to various physiological stimuli and can be measured using heart-rate variability (HRV). It is unclear how these mechanisms operate during pregnancy, particularly related to exercise. We examined BRG and HRV prior to, during, and following prenatal exercise. Forty-three pregnant ( $\mathrm{n}=10$ first trimester [TM1], $\mathrm{n}=17$ second trimester [TM2]; $\mathrm{n}=16$ third trimester [TM3]) and 20 non-pregnant (NP) women underwent an incremental peak exercise test. Beat-by-beat blood pressure (photoplethysmography) and heartrate (lead II ECG) were measured throughout. BRG (slope of the relationship between fluctuations in systolic blood pressure and R-R interval) and HRV (root mean square of the successive differences; RMSSD) were assessed at rest, during steady-state exercise (EX), and during active recovery. BRG decreased with gestation and was lower in TM3 compared to NP (17.9 \pm 6.9 vs $24.8 \pm 7.4 \mathrm{~ms} / \mathrm{mmHg}, p=0.017)$. BRG was reduced during EX in all groups. Resting HRV (RMSSD) also decreased with gestation and was lower in TM3 compared to NP ( $29 \pm 17$ vs $48 \pm 20 \mathrm{~ms}, p<0.001)$. RMSSD was blunted during EX for all groups compared to REST. During active recovery, RMSSD was further blunted compared to EX in NP, but not during pregnancy (TM1, TM2, and TM3). Compared to non-pregnant controls, pregnant women had lower BRG and HRV at rest, but comparable cardioautonomic control during both exercise and active recovery following peak exercise.

Key words: Arterial baroreflex, heart rate variability, exercise recovery, autonomic nervous system, pregnancy 


\section{Introduction}

Pregnancy is a physiological stressor which is associated with significant adaptations to the cardioautonomic nervous system (Davenport et al. 2016; Usselman et al. 2015). As pregnancy progresses, the balance between the sympathetic and parasympathetic nervous system activity shifts towards lower parasympathetic control. This balance can be assessed using the non-invasive techniques of heart rate variability (HRV), cardiac time interval and cardiovagal baroreflex gain (BRG) (Malik 1998; Allen et al. 2007; Picard et al. 2009).

As pregnancy progresses and sympathetic activation increases, resting heart rate variability declines, suggesting blunted parasympathetic control. This includes increased normalized high-frequency power and both decreased Root Mean Squared of Successive Differences (RMSSD) and Standard Deviation of N-N Intervals (SDNN) (Carpenter et al. 2017a; Chamchad et al. 2007; Kuo et al. 2000; Speranza et al. 1998). Concurrently, the duration of ventricular depolarization and repolarization, electrocardiographically measured with the QT interval, declines (Carpenter et al. 2015). Other cardiac time intervals are not well explored in pregnancy. Acute drops in blood pressure are met with a reflexive increase in heart rate via the cardiovagal baroreflex to maintain arterial blood pressure. Though a few studies suggest otherwise (Leduc et al. 1991; Seligman 1971), most studies suggest that during pregnancy, the cardiovagal baroreflex has a decreased gain in response to spontaneous changes in blood pressure (Blake et al. 2000; Carpenter et al. 2017b; Greenwood et al. 2001; Lucini et al. 1999; Silver et al. 2001; Visontai et al. 2002).

Similar cardioautonomic responses to those observed in pregnancy (i.e., decreased cardiovagal baroreflex gain and heart rate variability) have been identified in response to acute exercise in non-pregnant adults. Studies have shown that exercise is associated with sympathetic 
activation and a blunted HRV (Carpenter et al. 2017a). Also similar to the cardiac time interval changes observed at rest in pregnancy, QT interval shortens with progressive exercise in healthy individuals (Lewis and Short 2010). In conjunction with sympathetic activation, BRG is blunted and the baroreflex is reset to a higher operating heart rate and blood pressure (Sheriff 2006). Following exercise in non-pregnant adults the mechanisms regulating blood pressure are altered by a drop is systemic vascular resistance, resulting in a higher incidence of post-exercise hypotension (Senitko et al. 2002). This hypotension is due to persistent vasodilation which is not appropriately compensated for by an increased cardiac output.

During pregnancy, despite increases in both heart rate and stroke volume, systemic vasodilation ultimately leads to a decrease in total peripheral resistance and mean arterial pressure (Davenport et al. 2016). Along with a decrease in total peripheral resistance, pregnant women have blunted cardioautonomic control at rest, which may lead to an increased risk of hypotension, pre-syncope or fainting during and following exercise. While the cardiovascular responses to exercise during pregnancy have been broadly reviewed previous (Davenport et al. 2016), the cardioautonomic control of pregnant women during and following exercise throughout gestation remains unclear.

Therefore, the purpose of this study was to assess autonomic nervous system control and blood pressure regulation at rest, during light exercise, and during active recovery following an acute bout of peak exercise in pregnant $\left(1^{\text {st }}, 2^{\text {nd }}\right.$ and $3^{\text {rd }}$ trimester $)$ and non-pregnant women. We hypothesized that compared to non-pregnant women, gestation would be associated with an attenuation of the cardiovagal baroreflex, heart rate variability and cardiac time intervals at rest and during exercise. We further hypothesized that pregnant women would have a blunted 
cardioautonomic recovery following exercise, as indicated by continued attenuation of cardiovagal baroreflex gain and heart rate variability during the active recovery period.

\section{Methods}

\section{Ethical approval}

This test protocol was approved by the Health Research Ethics Board at the University of Alberta (Approval \#: Pro00040722) on October 25 $5^{\text {th }}, 2013$ and conformed to the standards set by the latest revision of the Declaration of Helsinki, except for registration in a database. All participants provided both verbal and written informed consent prior to participation in this study.

\section{Participants}

As part of a larger study, 38 participants completed testing at least once, to be included in one of the four groups (non-pregnant, first trimester, second trimester, or third trimester). A subset of participants were followed-up and completed repeated testing in additional trimesters, and were thus included in more than one group. As such, tests were completed on 20 non-pregnant women, 10 women in the first trimester (range: 10-12 weeks gestation), 17 women in the second trimester (range: 20-27 weeks gestation), and 16 women in the third trimester (range: 30-37 weeks gestation. A thorough breakdown of the testing scheme for participants is provided in the online supplement to this manuscript ${ }^{1}$. Briefly, 15 non-pregnant women were independently tested, one woman tested when non-pregnant was followed up in the $2^{\text {nd }}$ trimester, one woman tested when non-pregnant was followed up in the $3^{\text {rd }}$ trimester, and three women tested when non-pregnant were followed up in all 3 trimesters following conception. Two women were independent tested in the $1^{\text {st }}$ trimester, one initially tested in the $1^{\text {st }}$ trimester was followed up in 
the $2^{\text {nd }}$ trimester, one initially tested in the $1^{\text {st }}$ trimester was followed up in the $3^{\text {rd }}$ trimester, and three initially tested in the $1^{\text {st }}$ trimester were followed up in both the $2^{\text {nd }}$ and $3^{\text {rd }}$ trimester. Three participants were independently tested in the $2^{\text {nd }}$ trimester, while six participants initially tested in the $2^{\text {nd }}$ trimester were followed up in the $3^{\text {rd }}$ trimester. Finally, two participants were independently tested in the $3^{\text {rd }}$ trimester. Gestational age was calculated from the first day of the last menstrual period. None of the participants reported a history of gestational diabetes, gestational hypertension, or preeclampsia. Non-pregnant women were tested during the early follicular phase of the menstrual cycle, with the exception of those who were taking hormonal contraceptives where cycling had ceased (Nuva Ring, $n=1$; Min Ovral, $n=1$ Yasmin, $n=1$; Yaz, $\mathrm{n}=1$ ); these women were tested at their convenience. Pregnant women received medical clearance from their health care provider prior to exercise testing (PARMed-X 2013). Nonpregnant women completed the Physical Activity Readiness Questionnaire (PAR-Q) to prescreen for potential contraindications to exercise (Par-Q \& You 2002).

\section{Experimental protocol}

Participants arrived at the laboratory at 8:00am following a 12-hour fast, and having abstained from caffeine, alcohol, and strenuous exercise for 12 hours. Upon arrival at the lab, participants were fed a light, standardized meal, including a multigrain bagel with jam and orange juice. Weight was measured with a standard calibrated scale, and height was measured with a stadiometer prior to the start of testing. Participants were seated on a recumbent cycle

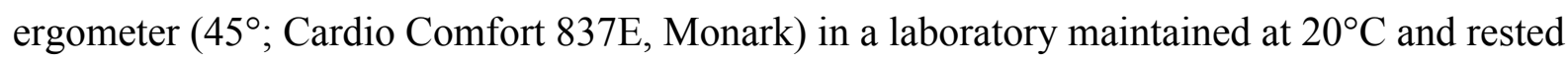
for a minimum of 20 minutes before beginning the exercise protocol. Following a five-minute inactive baseline (REST), participants cycled for five minutes at 25 watts $(\mathrm{W})$, maintaining a pedal rate of $50 \mathrm{rpm}(\mathrm{EX})$. Following the five-minute warm-up, pedal rate was maintained at 50 
rpm, while work rate was increased $25 \mathrm{~W} /$ min until volitional fatigue. Perceived exertion was recorded every second increment during exercise, using a Borg scale (Borg 1970). Upon reaching volitional fatigue, participants underwent a five-minute active recovery period back at $25 \mathrm{~W}(\mathrm{POST})$.

\section{Instrumentation}

Participants were instrumented with a respiratory apparatus consisting of a nose clip, mouthpiece, disposable bacteriological filter, and a heated $\left(37^{\circ} \mathrm{C}\right)$ respiratory flow head (ADInstruments; ADI, MLT1000L, Colorado Springs, CO, USA). Respiratory flow was measured by the pneumotachometer (Hans Rudolph Inc., MLT3819H-V, Shawnee, KS, USA) and spirometer amplifier (ADI, FE141, Colorado Springs, CO, USA). The respiratory flow head was calibrated daily using a 3L calibration syringe (Hans Rudolph Inc., 5530, Shawnee, KS, USA).

A continuous electrocardiogram (ECG; Lead II) was recorded throughout testing. Blood pressure was measured continuously using photoplethysmography (Finometer; Finapres Medical Systems, Amsterdam, The Netherlands) and later calibrated using baseline blood pressure values obtained using manual mercury sphygmomanometry. Calibrated pressure waveforms were analyzed to determine beat-by-beat mean arterial (MAP), systolic (SBP), and diastolic (DBP) blood pressures. All signals were recorded at a sampling frequency of $1000 \mathrm{~Hz}$.

\section{Data and statistical analyses}

R-R interval (RRI) and heart-rate (HR) were extracted on a beat-by-beat basis from the ECG tracing. HRV, BRG, and additional electrocardiogram (ECG) analyses (see below) were completed on data extracted from the last four minutes of three stages during the incremental 
peak exercise test: the inactive baseline (REST), the $25 \mathrm{~W}$ warm-up (mild exercise; EX) and the post-exercise active recovery $(25 \mathrm{~W})$ period (POST).

HRV was analyzed using commercially available software (ADI, MLS310/8 HRV, Colorado Springs, CO, USA). Frequency domain analyses included spectrum analysis of the very-low frequency (VLF; 0-0.04 Hz), low-frequency (LF; 0.04-0.15 Hz), and high-frequency (HF; 0.15-0.40 Hz) bands. The HRV module for this software uses the Lomb Periodogram nonparametric method for spectral analysis, with a default frequency number of 500. Temporal domain analyses included the standard deviations of the deviations between successive N-N intervals (SDNN) and Root Mean Square of Successive Differences (RMSSD). Total power was calculated as the variance of all normal-to-normal (NN) intervals. The ratio of Low Frequency to High Frequency Power (LF/HF) was also used.

Spontaneous cardiovagal baroreflex gain was determined by the sequence method (Blaber et al. 1995; Parlow et al. 1995; Usselman et al. 2015; Smyth et al. 1969) using an ADInstruments Chart Software (version 8.1.5) algorithm. Sequences of three or more consecutive beats exhibiting concurrent changes in systolic blood pressure and R-R interval (both rising or both falling) were identified. Sequences with rising blood pressure were classified as UP events, while sequences with falling blood pressure were classified as DOWN events.

Mean BRG was taken as the average of these two classifications. Identified sequences were then visually confirmed by a trained observer prior to analyses. On the basis of evidence that the R-R interval is typically modulated within the same cardiac cycle (Pickering and Davies 1973), data sets were analyzed using a lag 0 (same beat) criterion (Blaber et al. 1995). This methodology is consistent with previous research (Sobierajski et al. 2018; Steinback et al. 2009). The mean slope of identified sequences was taken to represent cardiovagal baroreflex gain, and only sequences 
with an $\mathrm{r}^{2}>0.8$ were included in the analysis. The cardiovagal baroreflex set point was determined as the prevailing average R-R interval and systolic blood pressure of the sequences.

ECG analysis was performed using spectral analysis software (ADI, MLS360/8 ECG Analysis, Colorado Springs, CO, USA). Ectopic beats and noise-contaminated waveforms were automatically detected by the software and were later confirmed and removed by a trained observer. Cardiac time intervals were defined as follows: P duration (atrial depolarization), PR Interval (atrial depolarization onset to ventricular depolarization onset), QRS Interval (ventricular depolarization), QT Interval (ventricular depolarization and repolarization), QTc (rate-corrected QT time), JT interval (ventricular repolarization), and Tpeak to Tend interval (ending of ventricular repolarization).

Baseline hemodynamics and respiratory characteristics were compared between groups using One Way ANOVA. Baroreflex gain, heart rate variability measures and cardiac time intervals analyzed during REST, EX, and POST were compared between groups using Two Way Repeated Measures ANOVA, Group (NP, TM1, TM2, TM3) by State (Rest, Exercise, Recovery), One Factor Repetition - Holm-Sidak Multiple Comparison Method. For all statistical analyses, alpha was set at 0.05. All statistical analyses were performed using SigmaPlot (version 13.0; Systat Software, San Jose, CA, USA). Data are presented as means \pm SD.

\section{Results}

\section{Anthropometrics and hemodynamics}

Pregnant women were of similar age and pre-pregnancy body mass index (BMI) as the non-pregnant women (Table 1). Physiological adaptations to pregnancy were observed including an elevation in heart rate with increasing gestation compared to non-pregnant women. Pregnant 
women demonstrated a drop in arterial pressure in TM2 which returned to NP levels in TM3 (Table 1). No significant differences in peak $\mathrm{VO}_{2}$ and peak power output at volitional fatigue during the graded exercise test were observed between groups (Table 2).

\section{Heart rate variability}

At REST, indices of HRV (R-R Interval, Standard Deviation of N-N Intervals, Total Power) were significantly lower in all pregnant groups compared to the non-pregnant group (Table 3). Also, RMSSD was lower in the $3^{\text {rd }}$ trimester $(29 \pm 17 \mathrm{~ms})$ than in the non-pregnant group (48 $\pm 20 \mathrm{~ms}, p<0.001)$. For all groups, $\mathrm{R}-\mathrm{R}$ interval significantly decreased from rest to the light steady state exercise period. R-R intervals continued to decrease during the graded exercise protocol across the four groups, were lowest at volitional fatigue (Table 2), and remained below resting values during the active recovery period. The Standard Deviation of N-N Intervals decreased from REST to EX for all groups and returned to resting levels during active recovery for the pregnant groups but not the non-pregnant group. RMSSD decreased from REST to EX in all groups, and further decreased from EX to POST in the non-pregnant group, but not in the pregnant groups. For all groups, Total Power and High Frequency Power were reduced during EX and POST compared to REST. Low Frequency Power was significantly reduced from REST to EX in the non-pregnant, $2^{\text {nd }}$ trimester, and $3^{\text {rd }}$ trimester groups. Low Frequency Power was also reduced from REST to POST in all groups. The Low Frequency/High Frequency ratio was higher during EX than REST in both the non-pregnant and $3^{\text {rd }}$ trimester group. Further, the ratio was higher during POST than REST in all groups other than the $1^{\text {st }}$ trimester group. The total change in RMSSD from REST to POST was significantly lower in TM3 than in NP $(-21.6 \pm 14.8$ vs $-39.3 \pm 19.8 \mathrm{~ms}, p=0.018$, Figure 1$)$.

\section{Baroreflex}


At rest, Up, Down, and Mean BRG were significantly lower in the $3^{\text {rd }}$ trimester women relative to the non-pregnant group $(17.9 \pm 6.9$ vs $24.8 \pm 7.4 \mathrm{~ms} / \mathrm{mmHg}, \mathrm{p}=0.017$; Table 4$)$. During steady stage exercise, there were significantly blunted Up, Down, and Mean BRG for all groups, which were blunted further during active recovery following peak exercise in the non-pregnant and $2^{\text {nd }}$ trimester groups. The amount of blunting from REST to EX (delta) was similar between groups (Figure 2). However, Mean BRG in the $3^{\text {rd }}$ trimester group was blunted significantly less from the graded exercise test (from EX to POST) than in the non-pregnant group $(-3.1 \pm 4.2$ vs $8.8 \pm 8.7 \mathrm{~ms} / \mathrm{mmHg}, p=0.042)$. Additionally, the total change in Mean BRG from REST to POST was significantly smaller in the $3^{\text {rd }}$ trimester women than in the non-pregnant group $(-13.0$ \pm 6.6 vs $-20.7 \pm 7.9 \mathrm{~ms} / \mathrm{mmHg}, p=0.028)$.

\section{Cardiac time intervals}

Cardiac time intervals are presented in Table 5. QRS interval increased progressively from rest, to steady state exercise to active recovery following the graded exercise test in the non-pregnant group. For all other groups, QRS interval was only elevated POST, when compared to REST. At REST, QT and JT intervals were significantly reduced in the $3^{\text {rd }}$ trimester group relative to the non-pregnant group; QT shortening was no longer significant after correcting for the duration of the cardiac cycle (QTc). Resting QT interval was reduced during steady state exercise in all groups other than the $3^{\text {rd }}$ trimester. Further, due to the rising difficulty of the graded exercise test, QT interval was significantly lower during the active recovery period following volitional fatigue than during the light steady-state exercise warm-up in all groups. Resting rate-corrected QT interval (QTc) was elevated during both during steady state exercise and following the graded exercise test in the non-pregnant group and elevated exclusively following the graded exercise test in the $3^{\text {rd }}$ trimester group. For all groups, resting JT interval 
was reduced during the steady-stage EX period, and further reduced during the graded test recovery period.

\section{Discussion}

Our data demonstrate a pregnancy-related blunting of the cardio-autonomic system with gestation and an exercise-related blunting in HRV in all groups. However, during active recovery following peak exercise pregnant women's cardioautonomic control appears to have been less attenuated than that of non-pregnant controls.

Our observations of diminished resting HRV (SDNN and RMSSD) with gestation, indicating parasympathetic control blunting, are consistent with previous research (Carpenter et al. 2015; Chamchad et al. 2007; Lucini et al. 1999; Speranza et al. 1998). This was matched with a marked reduction in total power, indicating sympathetic dominance (Task Force of the European Society of Cardiology and North American Society of Pacing and Electrophysiology 1996). Thus, it appears there's a marked increase in sympathetic control with gestation. Reductions in HRV are linked to unfavorable outcomes, such as obesity and diabetes (May et al. 2016). A shift in autonomic regulation from parasympathetic to sympathetic control, however, is essential to maintain proper blood flow to the growing conceptus during normal, healthy pregnancy. Thus, the observed shift in autonomic control is likely a natural gestational change.

Heart rate responses to exercise have prognostic power for cardiovascular morbidity and total mortality, and HRV provides a good insight into the cardiac vagal control of these responses (Nederend et al. 2016). Higher heart rate variability increases electrical stability of the heart (Hull et al. 1994). Our findings of lower Standard Deviation of N-N Intervals, RMSSD, and Total Power at rest in pregnancy are consistent with previous research (Carpenter et al. 
2017a; Carpenter et al. 2015; Avery et al. 2001). Interestingly, whether these differences persist into exercise appears to be unclear. Findings by Carpenter et al. (2015) showed that Total Power, High Frequency Power, Low Frequency Power, Standard Deviation of N-N Intervals and RMSSD remained lower during exercise in pregnancy. In contrast, findings by Avery et al. (2001) agreed that Low Frequency Power remained lower during sub-threshold exercise in pregnancy but showed that Total Power and High Frequency Power were similar between pregnant and non-pregnant women during exercise. Finally, we observed relatively comparable autonomic control during light exercise between groups. Indeed, while we used a fairly light recumbent cycling protocol, Carpenter et al. (2015) used a light stepping exercise protocol and Avery et al. (2001) used upright cycling at a higher intensity than us (60\% of ventilatory threshold), which may explain some of these discrepancies Our findings of comparable autonomic control during exercise across groups likely stems from the lower levels of parasympathetic modulation observed at rest during pregnancy, leading to a lower magnitude of parasympathetic withdrawal upon initiation of exercise during pregnancy (Avery et al. 2001).

Following peak exercise, most of the HRV measures suggested a continuation of sympathetic dominance into the active recovery period. An exception was that while SDNN remained lowered during active recovery in non-pregnant controls, a restoration of resting SDNN was seen in the pregnant groups. However, RMSSD is traditionally preferred over SDNN as a time domain measure in exercise settings due to the shorter duration of recording time in this setting (Buchheit 2014) And the lower sensitivity to breathing patterns that RMSSD has (Penttila et al. 2001). RMSSD was further blunted during active recovery in the non-pregnant controls and did not significantly change from light exercise during the active recovery period following peak exercise in the pregnant groups. As such, like what was seen during light exercise, based on 
higher resting sympathetic modulation, pregnant women likely experience a lower magnitude of sympathetic activation during peak exercise and perhaps experienced less vagal stress. Since a decrease in RMSSD is indicative of a shift away from parasympathetic control, the assumption that pregnant women experienced less sympathetic activation is supported by the smaller decrease in RMSSD observed from rest to post-exercise recovery in late pregnancy compared to the non-pregnant group (Figure 1). Indeed, there is indication that sympathoadrenal responses to strenuous exercise are blunted during late pregnancy (Avery et al. 2001).

Previous research in non-pregnant adults has shown a positive relationship between BRG and HRV, indicating that changes in heart rate stemming from the cardiovagal baroreflex contribute to heart rate variability (Lucini et al. 2002). The blunting of resting BRG seen during late pregnancy in this study supports the findings of several previous studies (Blake et al. 2000; Carpenter et al. 2017b; Greenwood et al. 2001; Lucini et al. 1999; Silver et al. 2001; Visontai et al. 2002). This blunting is likely related to the vagal withdrawal and sympathetic activation observed at rest. In the current study, resting women in late pregnancy were less able to respond to acute changes in blood pressure than resting non-pregnant women. Blunted acute blood pressure regulation, such as that seen in pregnancy here, has been linked to orthostatic intolerance in non-pregnant populations (Custaud et al. 2002), so may represent a potential mechanism explaining reductions in blood pressure and increases in incidence of orthostatic intolerance in pregnancy. Additional research is needed to further assess the relationship between the blunting of BRG and orthostatic intolerance in pregnancy.

A gestation-associated blunting of acute blood pressure regulation was shown by a lower BRG in TM3 than NP. However, it is well documented that both an acute, as well as more prolonged periods of hypotension can be reached following exercise in non-pregnant populations 
(Charkoudian et al. 2003; Willie et al. 2011). This study is the first to examine cardiovagal baroreflex during both exercise and active recovery in pregnant women. The exercise-associated blunting of BRG seen across all gestational groups is consistent with previous studies involving non-pregnant populations (Bristow et al. 1971; Pickering et al. 1971). Despite a reduced baroreflex at rest, pregnant women were capable of reflexively controlling blood pressure during and following exercise. This supports previous findings of similar spontaneous baroreflex control between non-pregnant women and women in late pregnancy both above and below ventilatory threshold, despite having different baroreflex gain at rest (Avery et al. 2001). Further, this study adds that the similar spontaneous baroreflex control between pregnant and non-pregnant women observed during exercise persists in the recovery period following peak exercise.

Baroreflex gain was blunted less following peak exercise in pregnant relative to nonpregnant women, as shown by the persistent blunting seen in the non-pregnant women, and not the third trimester women. This is also reflected by the greater change in baroreflex gain observed in the non-pregnant women compared to the third trimester women from exercise to post (Figure 2). No differences in peak wattage or $\mathrm{VO}_{2}$ were seen between groups (Table 2), so the loss of gestation-associated differences in both baroreflex and heart rate variability observed at rest do not appear to be related to the intensity reached at volitional fatigue. Gestationassociated baroreflex blunting could be attributable to the higher cardiac output, plasma volume, and $\mathrm{RBC}$ volume seen in pregnancy. Overall, it appears that peak exercise had a more profound cardiovascular effect on non-pregnant women than those in late pregnancy. It is possible that since the baroreflex of those in late pregnancy is already blunted, further cardiovascular stress (i.e., exercise) enacts smaller effects than for those who have regular baroreflex function (i.e., non-pregnant women). Essentially, a floor effect brought on by the summation of these 
cardiovascular stressors may explain this difference. The likelihood of a floor effect in baroreflex gain is supported by the findings illustrated in Figure 2. The magnitude of blunting brought on by peak exercise was lower in late pregnancy than in non-pregnant women and as women in late pregnancy had an already blunted baroreflex gain at rest, may have a limited the ability for the baroreflex to further blunt as exercise intensity increased. Further investigation into the dynamic blood pressure regulation during pregnancy is warranted to better understand the implications of the relative leveling of baroreflex gain between pregnant and non-pregnant women.

Hemodynamic stress during pregnancy has been suggested to lead to the generation of arrhythmias such as ventricular tachycardia and premature ventricular contraction (Nakagawa et al. 2004), but despite extensive hemodynamic changes, little is known about electrophysiological cardiac changes during pregnancy (Baumert et al. 2010). The observed reduction in QT interval, the duration of ventricular depolarization and repolarization, with advancing gestation supports previous findings. (Carpenter et al. 2015; Carpenter et al. 2017a), although the previous studies showed that QT interval was altered as early as the first trimester, these studies agree that ratecorrected QT (QTc) is not altered during pregnancy (Baumert et al. 2010). Changes in cardiac time interval in response to exercise were as expected. Similar to what has been documented in healthy populations previously, QT Interval was reduced in non-pregnant, $1^{\text {st }}$ trimester, and $2^{\text {nd }}$ trimester groups. A reduction was not observed in the $3^{\text {rd }}$ trimester group, likely because QT Interval was already lower at rest. Rises in QT interval both during and after exercise may indicate myocardial ischemia (Egloff et al. 1987; Watanabe et al. 1998) and be related to risk of sudden death following exercise (Sundaram et al. 2008). However, no abnormal changes like such were observed during and following exercise in the current study. Other cardiac time intervals were included to complement QT interval and provide additional indication into the 
intrinsic cardiac changes that might occur during prenatal exercise. Prolongation of the PR Interval and QRS interval during and following exercise are associated with higher risk of atrioventricular block (Nieminen et al. 2010), and ventricular arrhythmias (Berntsen et al. 1995), respectively. As such, the observed constancy of PR interval throughout the current study protocol is favourable. A small rise in QRS interval was observed during the recovery period. However, the magnitude of that rise was well below the $15 \mathrm{~ms}$ change typically used for predictive purposes (Berntsen et al. 1995). Overall, the cardiac time interval changes observed in the current study were as expected and identified no negative electrophysiological changes during light exercise and during active recovery following peak exercise.

\section{Limitations}

Admittedly, longitudinal analyses would provide better insight than inter-subject analyses. Repeated measures were performed on a subset of participants in this study at all three pregnant groups, but the small number $(\mathrm{n}=6)$ of those participants did not lend itself to analysis restricted to longitudinal data. And although this study included relatively small sample sizes from each group, groups were matched for age and BMI and represent the average for healthy pregnancies. In this study, the spontaneous method for calculating cardiac baroreflex gain was employed, as opposed to the "gold standard" modified Oxford method. The modified Oxford method examines responses to a wider range of blood pressures through pharmacological means, but this method is not ideally suited to pregnant women (Jarvis et al. 2012). Further, spontaneous baroreflex gain measures have been validated against the modified Oxford method previously (Hart et al. 2010). Thus, although interpretation of cardiovagal baroreflex gain is limited to that around the operating point, this has relevance for immediate homeostatic blood pressure control. In the current study, cardiorespiratory conditioning status of the participants was not statistically accounted for, which can influence cardiovagal baroreflex gain, heart rate variability, and 
electrocardiogram characteristics (Carpenter et al. 2017a; Carpenter et al. 2017b). However, both peak wattage and VO2 were similar between groups (Table 2), and thus makes it less likely that significant differences in training status exist between groups. It is noteworthy that although nonsignificant, pregnant women had lower peak wattage, which could influence interpretations of the recovery period. It is possible that the small difference in wattage could influence the coinciding cardioautonomic measures in the recovery period. The alternative to using peak exercise would have been to use a standardized submaximal load for all participants. However, a standardized wattage would potentially result in differences in relative intensities of exercise for each participant. In the model used in the current study, peak wattage was targeted to assess recovery from peak exercise. This resulted in similar percentages of age-predicted max heart rate (220-age) being reached in the four groups (Table 2).

Further, due to the nature of the protocol, exercise-related assessment of the measures was limited to the light exercise portion of the protocol before the incremental test, as well as the active recovery period following the test. The cardiovascular responses to exercise during pregnancy have been reviewed previously (Davenport et al. 2016), and our focus was to understand post-exercise recovery during pregnancy. An alternative would have been to evaluate a resting recovery period following the incremental exercise test to more directly compare to initial resting values. However, best practice, as recommended by the CSEP Clinical Practice Guidelines for Exercise in Pregnancy and the Postpartum Period, is to follow aerobic exercise with a 5-minute cool-down, hence the reason for the active recovery chosen in the current study.

Lastly, the inclusion of a subset of the participants in more than one group complicates our interpretation. This data structure is most akin to repeated cross-sectional data captured in cohort studies. To assess the limitation of this data structure, results from the major variables in 
this study (baroreflex gain and heart rate variability) were compared to a data subset including only participants measured at a single timepoint (non-repeat measure participants). The results of the comparisons are provided in the online supplement to this manuscript ${ }^{2}$. As seen, both cardiovagal baroreflex gain and RMSSD were blunted in the $3^{\text {rd }}$ trimester relative to the nonpregnant group in the subset analysis. Further, there are no significant differences in BRG and RMSSD observed when comparing the non-pregnant and $3^{\text {rd }}$ trimester groups in both exercise and recovery periods. This agrees with the results found with the original ANOVAS, which included all the tests (including repeated measures ones). Further, graphical illustrations of the changes participants underwent between conditions are provided in the online supplement to this manuscript ${ }^{3}$. This graph shows the values of participants measures more than once follow the trends of the participants measured at a single timepoint, as well as the group means. Overall, the inclusion of these repeated measures participants likely doesn't influence the results and interpretations.

\section{Conclusion}

In the present study, pregnancy was associated with a progressive blunting of resting parasympathetic control (heart rate variability) and blood pressure regulation (baroreflex gain). Despite these changes, pregnant women exhibited similar abilities to respond to fluctuations in blood pressure and control heart rate during and following exercise compared to non-pregnant women. In fact, pregnant women's cardioautonomic control was less affected during recovery following peak exercise. These data provide reassurance that pregnant women are able to adequately recover following acute exercise.

\section{Conflict of interest statement}

The authors declare no conflicts of interest.

${ }^{2}$ see supplementary table $\mathrm{S} 2$

${ }^{3}$ see supplementary figures $\mathrm{S} 1$ and $\$ 2$ 


\section{Acknowledgments}

GP was supported by a Women and Children's Health Research Institute Summer Studentship. PW was supported by a Women and Children's Health Research Institute Summer Studentship and Alberta Diabetes Institute Summer Studentship. MD is supported by a National New Investigator Award from the Heart \& Stroke Foundation of Canada and Health Canada Advancing Women's Heart Health Research Initiative. This research has been funded by the generous supporters of the Lois Hole Hospital for Women through the Women and Children's Health Research Institute.

\section{References}

Allen, J.J., Chambers A.S., and Towers D.N. 2007. The many metrics of cardiac chronotropy: a pragmatic primer and a brief comparison of metrics. Biol. Psychol. 74(2): 243-262. doi:10.1016/j.biopsycho.2006.08.005. PMID:17070982.

Avery, N.D., Wolfe L.A., Amara C.E., Davies G.A., and McGrath M.J. 2001. Effects of human pregnancy on cardiac autonomic function above and below the ventilatory threshold. J. Appl. Physiol. 90(1): 321-328. doi:10.1152/jappl.2001.90.1.321. PMID:11133925.

Baumert, M., Seeck A., Faber R., Nalivaiko E., and Voss A. 2010. Longitudinal changes in QT interval variability and rate adaptation in pregnancies with normal and abnormal uterine perfusion. Hypertens. Res. 33(6): 555-560. doi:10.1038/hr.2010.30. PMID:20224570. 
Berntsen, R.F., Gjestvang F.T., and Rasmussen K. 1995. QRS prolongation as an indicator of risk of ischemia-related ventricular tachycardia and fibrillation induced by exercise. Am. Heart J. 129(3): 542-548. doi:10.1016/0002-8703(95)90283-X. PMID:7872186.

Blaber, A.P., Yamamoto Y., and Hughson R.L. 1995. Methodology of spontaneous baroreflex relationship assessed by surrogate data analysis. Am. J. Physiol. 268(4 Pt 2): H1687. doi:10.1152/ajpheart.1995.268.4.H1682. PMID:7733371.

Blake, M.J., Martin A., Manktelow B.N., Armstrong C., Halligan A.W., Panerai R.B., et al. 2000. Changes in baroreceptor sensitivity for heart rate during normotensive pregnancy and the puerperium. Clin. Sci. 98(3): 259-268. doi:10.1042/cs0980259. PMID:10677383.

Borg, G. 1970. Perceived exertion as an indicator of somatic stress. Scand. J. Rehabil. Med. 2(2): 92-98. PMID:5523831.

Bristow, J., Brown J., E, Cunningham D., Howson M., Petersen E., Pickering T., et al. 1971. Effect of Bicycling on the Baroreflex Regulation of Pulse Interval. Circ. Res. 28(5): 582-592. doi:10.1161/res.28.5.582.

Buchheit, M. 2014. Monitoring training status with HR measures: do all roads lead to Rome? Front. Physiol. 5 73. doi:10.3389/fphys.2014.00073. doi:10.3389/fphys.2014.00073. PMID:24578692.

Carpenter, R.E., D'Silva L.A., Emery S.J., Uzun O., Rassi D., and Lewis M.J. 2015. Changes in heart rate variability and QT variability during the first trimester of pregnancy. Physiol. Meas. 36(3): 531-545. doi:10.1088/0967-3334/36/3/531. PMID:25690105. 
Carpenter, R.E., Emery S.J., Uzun O., Rassi D., and Lewis M.J. 2017a. Influence of antenatal physical exercise on heart rate variability and QT variability. J. Matern. Fetal Neonatal Med. 30(1): 79-84. doi:10.3109/14767058.2016.1163541. PMID:27023345.

Carpenter, R.E., Emery S.J., Uzun O., Rassi D., and Lewis M.J. 2017b. Influence of physical exercise on baroreceptor sensitivity during pregnancy. J. Matern. Fetal Neonatal Med. 30(5): 514-519. doi:10.1080/14767058.2016.1179275. PMID:27098455.

Chamchad, D., Horrow J.C., Nakhamchik L., and Arkoosh V.A. 2007. Heart rate variability changes during pregnancy: an observational study. Int. J. Obstet. Anesth. 16(2): 106-109. doi:10.1016/j.ijoa.2006.08.008. PMID:17270423.

Charkoudian, N., Halliwill J.R., Morgan B.J., Eisenach J.H., and Joyner M.J. 2003. Influences of hydration on post-exercise cardiovascular control in humans. J. Physiol. 552(Pt 2): 635-644. doi:10.1113/jphysiol.2003.048629. PMID:14561843.

Custaud, M.A., de Souza Neto, E P, Abry P., Flandrin P., Millet C., Duvareille M., et al. 2002. Orthostatic tolerance and spontaneous baroreflex sensitivity in men versus women after 7 days of head-down bed rest. Auton. Neurosci. 100(1-2): 66-76. doi:10.1016/S1566-0702(02)00132-7. PMID:12422962.

Davenport, M.H., Skow R.J., and Steinback C.D. 2016. Maternal Responses to Aerobic Exercise in Pregnancy. Clin. Obstet. Gynecol. 59(3): 541-551. doi:10.1097/GRF.0000000000000201. PMID:27042798. 
Egloff, C., Merola P., Schiavon C., Schiavinato M.L., Modena F., Stritoni P., et al. 1987. Sensitivity, specificity and predictive accuracy of Q wave, QX/QT ratio, QTc interval and ST depression during exercise testing in men with coronary artery disease. Am. J Cardiol. 60(13): 1006-1008. doi:10.1016/0002-9149(87)90342-0. PMID:3314455.

Greenwood, J.P., Scott E.M., Stoker J.B., Walker J.J., and Mary D.A. 2001. Sympathetic neural mechanisms in normal and hypertensive pregnancy in humans. Circulation. 104(18): 2200-2204. doi:10.1161/circ.104.18.2200. PMID:11684631.

Hart, E.C., Joyner M.J., Wallin B.G., Karlsson T., Curry T.B., and Charkoudian N. 2010. Baroreflex control of muscle sympathetic nerve activity: a nonpharmacological measure of baroreflex sensitivity. Am J Physiol Heart Circ Physiol 298(3): 816. doi:10.1152/ajpheart.00924.2009. PMID:20008270.

Hull, S.S., Vanoli E., Adamson P.B., Verrier R.L., Foreman R.D., and Schwartz P.J. 1994. Exercise training confers anticipatory protection from sudden death during acute myocardial ischemia. Circulation. 89(2): 548-552. PMID:8313542.

Jarvis, S.S., Shibata S., Bivens T.B., Okada Y., Casey B.M., Levine B.D., et al. 2012. Sympathetic activation during early pregnancy in humans. J. Physiol. 590(15): 3535-3543. doi:10.1113/jphysiol.2012.228262. PMID:22687610.

Kuo, C.D., Chen G.Y., Yang M.J., Lo H.M., and Tsai Y.S. 2000. Biphasic changes in autonomic nervous activity during pregnancy. Br. J. Anaesth. 84(3): 323-329. doi:10.1093/oxfordjournals.bja.a013433. PMID:10793590. 
Leduc, L., Wasserstrum N., Spillman T., and Cotton D.B. 1991. Baroreflex function in normal pregnancy. Am. J. Obstet. Gynecol. 165(4 Pt 1): 886-890. doi:10.1016/0002-9378(91)90433-R. PMID:1951548.

Lewis, M.J., and Short A.L. 2010. Exercise and cardiac regulation: what can electrocardiographic time series tell us? Scand. J. Med. Sci. Sports. 20(6): 794-804. doi:10.1111/j.1600-0838.2010.01150.x. PMID:20561274.

Lucini, D., Guzzetti S., Casiraghi S., and Pagani M. 2002. Correlation between baroreflex gain and 24-h indices of heart rate variability. J. Hypertens. 20(8): 1625-1631. PMID:12172325.

Lucini, D., Strappazzon P., Dalla Vecchia L., Maggioni C., and Pagani M. 1999. Cardiac autonomic adjustments to normal human pregnancy: insight from spectral analysis of R-R interval and systolic arterial pressure variability. J. Hypertens. 17(12 Pt 2): 1899-1904. PMID:10703887.

Malik, M. 1998. Heart rate variability. Curr. Opin. Cardiol. 13(1): 36-44. PMID:9559255.

May, L.E., Knowlton J., Hanson J., Suminski R., Paynter C., Fang X., et al. 2016. Effects of Exercise During Pregnancy on Maternal Heart Rate and Heart Rate Variability. PM R. 8(7): 611617. doi:10.1016/j.pmrj.2015.11.006. PMID:26603201.

Nakagawa, M., Katou S., Ichinose M., Nobe S., Yonemochi H., Miyakawa I., et al. 2004.

Characteristics of new-onset ventricular arrhythmias in pregnancy. J. Electrocardiol. 37(1): 4753. doi:10.1016/j.jelectrocard.2003.10.007. PMID:15132369. 
Nederend, I., Schutte N.M., Bartels M., Ten Harkel A.D., and de Geus E.J. 2016. Heritability of heart rate recovery and vagal rebound after exercise. Eur. J. Appl. Physiol. 116(11-12): 21672176. doi:10.1007/s00421-016-3459-y. PMID:27614881.

Nieminen, T., Verrier R.L., Leino J., Nikus K., Lehtinen R., Lehtimaki T., et al. 2010. Atrioventricular conduction and cardiovascular mortality: assessment of recovery PR interval is superior to pre-exercise measurement. Heart Rhythm, 7(6): 796-801. doi:10.1016/j.hrthm.2010.02.029. PMID:20188862.

Parlow, J., Viale J.P., Annat G., Hughson R., and Quintin L. 1995. Spontaneous cardiac baroreflex in humans. Comparison with drug-induced responses. Hypertension. 25(5): 10581068. doi:10.1161/hyp.25.5.1058. PMID:7737717.

Penttila, J., Helminen A., Jartti T., Kuusela T., Huikuri H.V., Tulppo M.P., et al. 2001. Time domain, geometrical and frequency domain analysis of cardiac vagal outflow: effects of various respiratory patterns. Clin. Physiol. 21(3): 365-376. doi:10.1046/j.1365-2281.2001.00337.x. PMID:11380537.

Picard, G., Tan C.O., Zafonte R., and Taylor J.A. 2009. Incongruous changes in heart period and heart rate variability with vagotonic atropine: implications for rehabilitation medicine. PM R. 1(9): 820-826. doi:10.1016/j.pmrj.2009.07.017. PMID:19769915.

Pickering, T.G., and Davies J. 1973. Estimation of the conduction time of the baroreceptorcardiac reflex in man. Cardiovasc. Res. 7(2): 213-219. doi:10.1093/cvr/7.2.213. PMID:4694105. 
Pickering, T.G., Gribbin B., Petersen E.S., Cunningham D.J., and Sleight P. 1971. Comparison of the effects of exercise and posture on the baroreflex in man. Cardiovasc. Res. 5(4): 582-586. doi:10.1093/cvr/5.4.582. PMID:5160463.

Seligman, S.A. 1971. Baroreceptor reflex function in pre-eclampsia. J. Obstet. Gynaecol. Br. Commonw. 78(5): 413-416. doi:10.1111/j.1471-0528.1971.tb00294.x. PMID:4326846.

Senitko, A.N., Charkoudian N., and Halliwill J.R. 2002. Influence of endurance exercise training status and gender on postexercise hypotension. J. Appl. Physiol. 92(6): 2368-2374. doi:10.1152/japplphysiol.00020.2002. PMID:12015349.

Sheriff, D.D. 2006. Baroreflex resetting during exercise: mechanisms and meaning. Am. J. Physiol. Heart Circ. Physiol. 290(4): H1407. doi:10.1152/ajpheart.01275.2005. PMID:16537789.

Silver, H.M., Tahvanainen K.U., Kuusela T.A., and Eckberg D.L. 2001. Comparison of vagal baroreflex function in nonpregnant women and in women with normal pregnancy, preeclampsia, or gestational hypertension. Am. J. Obstet. Gynecol. 184(6): 1189-1195. doi:10.1067/mob.2001.112871. PMID:11349187.

Smyth, H.S., Sleight P., and Pickering G.W. 1969. Reflex regulation of arterial pressure during sleep in man. A quantitative method of assessing baroreflex sensitivity. Circ. Res. 24(1): 109121. doi:10.1161/01.RES.24.1.109. PMID:4303309.

Sobierajski, F.M., Purdy G.M., Usselman C.W., Skow R.J., James M.A., Chari R.S., et al. 2018. Maternal physical activity is associated with improved blood pressure regulation during late pregnancy. Can. J. Cardiol. 34(4): 485-491. doi:10.1016/j.cjca.2018.01.021. PMID:29571429. 
Speranza, G., Verlato G., and Albiero A. 1998. Autonomic changes during pregnancy: assessment by spectral heart rate variability analysis. J. Electrocardiol. 31(2): 101-109. doi:10.1016/S0022-0736(98)90040-1. PMID:9588655.

Steinback, C.D., Salzer D., Medeiros P.J., Kowalchuk J., and Shoemaker J.K. 2009. Hypercapnic vs. hypoxic control of cardiovascular, cardiovagal, and sympathetic function. Am. J. Physiol. Regul. Integr. Comp. Physiol. 296(2): 402. doi:10.1152/ajpregu.90772.2008. PMID:19091913.

Sundaram, S., Carnethon M., Polito K., Kadish A.H., and Goldberger J.J. 2008. Autonomic effects on QT-RR interval dynamics after exercise. Am. J Physiol. Heart Circ. Physiol. 294(1): 490. doi:10.1152/ajpheart.00046.2007. PMID:17993603.

Task Force of the European Society of Cardiology, and North American Society of Pacing and Electrophysiology 1996. Heart rate variability: standards of measurement, physiological interpretation and clinical use. Circulation. 93(5): 1043-1065. doi:10.1161/circ.93.5.1043. PMID:8598068.

Usselman, C.W., Skow R.J., Matenchuk B.A., Chari R.S., Julian C.G., Stickland M.K., et al. 2015. Sympathetic baroreflex gain in normotensive pregnant women. J. Appl. Physiol. 119(5): 468-474. doi:10.1152/japplphysiol.00131.2015. PMID:26139215.

Visontai, Z., Lenard Z., Studinger P., Rigo J., and Kollai M. 2002. Impaired baroreflex function during pregnancy is associated with stiffening of the carotid artery. Ultrasound Obstet. Gynecol. 20(4): 364-369. doi:10.1046/j.1469-0705.2002.00820.x. PMID:12383319. 
Watanabe, T., Harumi K., Akutsu Y., Yamanaka H., Michihata T., Okazaki O., et al. 1998. Relation between exercise-induced myocardial ischemia as assessed by nitrogen-13 ammonia positron emission tomography and QT interval behavior in patients with right bundle branch block. Am. J Cardiol. 81(7): 816-821. doi: 10.1016/S0002-9149(98)00002-2. PMID:9555768.

Willie, C.K., Ainslie P.N., Taylor C.E., Jones H., Sin P.Y., and Tzeng Y.C. 2011. Neuromechanical features of the cardiac baroreflex after exercise. Hypertension. 57(5): 927-933. doi: 10.1161/HYPERTENSIONAHA.110.164616. PMID:21422379. 
Table 1. Descriptive characteristics of the participants.

\begin{tabular}{|c|c|c|c|c|}
\hline & $N P(n=20)$ & $T M 1(n=10)$ & $T M 2(n=17)$ & TM3 $(n=16)$ \\
\hline Age (years) & $28 \pm 6$ & $31 \pm 4$ & $31 \pm 4$ & $32 \pm 4$ \\
\hline Gestation (weeks) ${ }^{1}$ & $\mathrm{~N} / \mathrm{A}$ & $11 \pm 1$ & $24 \pm 2$ & $33 \pm 2$ \\
\hline Height (cm) & $167 \pm 7$ & $167 \pm 7$ & $166 \pm 7$ & $168 \pm 8$ \\
\hline Weight $(\mathrm{kg})$ & $66 \pm 11$ & $71 \pm 22$ & $71 \pm 14$ & $77 \pm 12$ \\
\hline Non/pre-pregnant BMI $\left(\mathrm{kg} / \mathrm{m}^{2}\right)$ & $23.8 \pm 3.4$ & $23.4 \pm 2.2$ & $23.0 \pm 2.8$ & $23.1 \pm 2.6$ \\
\hline Pregnant BMI $\left(\mathrm{kg} / \mathrm{m}^{2}\right)$ & $\mathrm{N} / \mathrm{A}$ & $25.7 \pm 8.1$ & $25.8 \pm 4.6$ & $27.1 \pm 2.2$ \\
\hline Heart rate (bpm) & $72 \pm 10$ & $80 \pm 11$ & $79 \pm 8$ & $84 \pm 11^{*}$ \\
\hline Mean arterial pressure $(\mathrm{mmHg})$ & $89 \pm 7$ & $87 \pm 8$ & $81 \pm 7^{*}$ & $84 \pm 6$ \\
\hline Systolic blood pressure $(\mathrm{mmHg})$ & $112 \pm 8$ & $113 \pm 10$ & $107 \pm 9$ & $108 \pm 5$ \\
\hline Diastolic blood pressure $(\mathrm{mmHg})$ & $74 \pm 7$ & $69 \pm 7$ & $66 \pm 7^{*}$ & $68 \pm 7$ \\
\hline Respiration Rate (breaths/min) & $18.4 \pm 2.6$ & $18.9 \pm 1.9$ & $18.0 \pm 3.3$ & $18.1 \pm 3.1$ \\
\hline
\end{tabular}

Values given as means \pm SD. Abbreviations: NP, non-pregnant; TM1, $1^{\text {st }}$ trimester; TM2, $2^{\text {nd }}$ trimester; TM3, $3^{\text {rd }}$ trimester.

${ }^{1}$ Significant differences between all groups $(P<0.05)$. *Significant difference vs NP $(P<0.05)$. 
Table 2. Ventilatory and power output parameters from a graded exercise test to volitional fatigue.

\begin{tabular}{|c|c|c|c|c|c|}
\hline & NP & TM1 & TM2 & TM3 & $p$ \\
\hline Resting VO2 (L/min) & $0.68 \pm 0.30$ & $0.69 \pm 0.18$ & $0.75 \pm 0.23$ & $0.87 \pm 0.18$ & 0.120 \\
\hline Peak VO2 (L/min) & $2.87 \pm 1.03$ & $2.73 \pm 1.04$ & $2.59 \pm 0.97$ & $3.30 \pm 1.26$ & 0.273 \\
\hline Peak Heart Rate (bpm) & $162 \pm 12$ & $161 \pm 9$ & $156 \pm 12$ & $154 \pm 11$ & 0.148 \\
\hline $\begin{array}{l}\% \text { of Age-Predicted HRmax } \\
\text { Reached (\%) }\end{array}$ & $84.7 \pm 5.4 \%$ & $85.2 \pm 5.1 \%$ & $82.6 \pm 6.1 \%$ & $82.0 \pm 5.9 \%$ & 0.344 \\
\hline Peak Wattage (W) & $173 \pm 28$ & $155 \pm 28$ & $154 \pm 21$ & $155 \pm 37$ & 0.151 \\
\hline
\end{tabular}

Values given as means \pm SD. Abbreviations: NP, non-pregnant; TM1, 1st trimester; TM2, 2nd trimester; TM3, 3rd trimester. 
Table 3. Heart rate variability data at rest, during light exercise, and during active recovery following peak exercise throughout pregnancy.

\begin{tabular}{|c|c|c|c|c|c|c|c|c|}
\hline & & $R R I(m s)$ & SDNN (ms) & $\begin{array}{c}R M S S D \\
(m s)\end{array}$ & $\begin{array}{c}\text { Total Power } \\
\left(\mu s^{2}\right)\end{array}$ & $\begin{array}{c}\text { LF Power } \\
\text { (nu) }\end{array}$ & $\begin{array}{c}\text { HF Power } \\
\text { (nu) }\end{array}$ & $L F / H F$ \\
\hline \multirow[t]{4}{*}{ REST } & $N P$ & $859 \pm 123$ & $61 \pm 19$ & $48 \pm 20$ & $3807 \pm 2991$ & $50 \pm 15$ & $47 \pm 14$ & $1.4 \pm 1.3$ \\
\hline & TM1 & $764 \pm 103^{*}$ & $44 \pm 16^{*}$ & $36 \pm 22$ & $2005 \pm 1675^{*}$ & $49 \pm 20$ & $49 \pm 20$ & $1.3 \pm 1.0$ \\
\hline & TM2 & $773 \pm 88^{*}$ & $44 \pm 13^{*}$ & $36 \pm 20$ & $2066 \pm 1504^{*}$ & $43 \pm 15$ & $56 \pm 15$ & $0.9 \pm 0.6$ \\
\hline & TM3 & $733 \pm 99^{*}$ & $44 \pm 20^{*}$ & $29 \pm 17^{*}$ & $1936 \pm 1457^{*}$ & $43 \pm 21$ & $55 \pm 21$ & $1.2 \pm 1.4$ \\
\hline \multirow[t]{4}{*}{$E X$} & $N P$ & $654 \pm 78 \dagger$ & $33 \pm 12 \dagger$ & $21 \pm 12 \dagger$ & $924 \pm 771 \dagger$ & $63 \pm 19 \dagger$ & $23 \pm 15 \dagger$ & $5.1 \pm 5.6 \dagger$ \\
\hline & TM1 & $631 \pm 89+$ & $30 \pm 12 \dagger$ & $18 \pm 11 \dagger$ & $665 \pm 717 \dagger$ & $53 \pm 19$ & $33 \pm 20 \dagger$ & $2.3 \pm 1.5$ \\
\hline & TM2 & $626 \pm 56 \dagger$ & $25 \pm 8 †$ & $15 \pm 9 \dagger$ & $555 \pm 460 \dagger$ & $57 \pm 22 \dagger$ & $30 \pm 20 \dagger$ & $3.5 \pm 3.3$ \\
\hline & TM3 & $629 \pm 65 \dagger$ & $24 \pm 10 \dagger$ & $13 \pm 9 \dagger$ & $596 \pm 709 \dagger$ & $65 \pm 21 \dagger$ & $30 \pm 21 \dagger$ & $5.9 \pm 9.5 \dagger$ \\
\hline \multirow[t]{4}{*}{ POST } & $N P$ & $500 \pm 60 † \ddagger$ & $49 \pm 18 † \ddagger$ & $9 \pm 6 † \ddagger$ & $393 \pm 417 \dagger$ & $70 \pm 19 \dagger$ & $16 \pm 9 \dagger$ & $6.3 \pm 4.8 \dagger$ \\
\hline & TM1 & $507 \pm 56 \dagger \ddagger$ & $47 \pm 21 \ddagger$ & $9 \pm 5 \dagger$ & $432 \pm 340 \dagger$ & $73 \pm 10 \dagger \ddagger$ & $20 \pm 7 \dagger$ & $4.1 \pm 1.8$ \\
\hline & TM2 & $518 \pm 51 † \ddagger$ & $42 \pm 15 \ddagger$ & $8 \pm 4 \dagger$ & $378 \pm 248 \dagger$ & $64 \pm 25 \dagger$ & $22 \pm 17 \dagger$ & $5.9 \pm 5.0 \dagger$ \\
\hline & TM3 & $528 \pm 47 \dagger \ddagger$ & $43 \pm 19 \ddagger$ & $7 \pm 4 \dagger$ & $495 \pm 442 \dagger$ & $74 \pm 18 \dagger$ & $21 \pm 15 \dagger$ & $6.2 \pm 4.6 \dagger$ \\
\hline
\end{tabular}

Values given as means \pm SD. Abbreviations: RRI, R-R interval; SDNN, standard deviation of consecutive R-R intervals; RMSSD, Root Mean Square of the Successive Differences; LF, low frequency; nu, normalized units; HF, high frequency; LF/HF, low frequency/high frequency ratio; REST, resting; EX, exercise; POST, active recovery; NP, non-pregnant; TM1, $1^{\text {st }}$ trimester; TM2, $2^{\text {nd }}$ trimester; TM3, $3^{\text {rd }}$ trimester. *Significant different from NP, within condition $(P<0.05)$. $†$ Significant difference from rest, same group $(P<0.05)$. $¥$ Significant difference from exercise, same group $(P<0.05)$. 
Table 4. Up, down, and mean baroreflex gain along with baroreflex setpoint (SBP, RRI) at rest, during light exercise, and during active recovery following peak exercise throughout pregnancy.

\begin{tabular}{|c|c|c|c|c|c|c|}
\hline & & Up (ms/mmHg) & Down (ms/mmHg) & Mean $(\mathrm{ms} / \mathrm{mmHg})$ & $S B P(m m H g)$ & $R R I(m s)$ \\
\hline \multirow[t]{4}{*}{ REST } & $N P$ & $28.7 \pm 10.7$ & $20.9 \pm 7.9$ & $24.8 \pm 7.4$ & $114.5 \pm 9.3$ & $852.6 \pm 122.1$ \\
\hline & TM1 & $21.5 \pm 10.1$ & $17.6 \pm 11.3$ & $19.6 \pm 10.4$ & $111.2 \pm 4.8$ & $762.2 \pm 103.6^{*}$ \\
\hline & TM2 & $26.3 \pm 10.2$ & $17.7 \pm 8.2$ & $22.0 \pm 8.7$ & $107.0 \pm 8.6$ & $767.0 \pm 82.6^{*}$ \\
\hline & TM3 & $22.0 \pm 8.0^{*}$ & $13.7 \pm 6.9^{*}$ & $17.9 \pm 6.9^{*}$ & $109.0 \pm 5.7$ & $725.7 \pm 94.0^{*}$ \\
\hline \multirow[t]{4}{*}{$E X$} & $N P$ & $14.5 \pm 9.7 \dagger$ & $11.1 \pm 8.2 \dagger$ & $12.8 \pm 8.8 \dagger$ & $124.8 \pm 9.3 \dagger$ & $653.9 \pm 79.1 \dagger$ \\
\hline & TM1 & $8.9 \pm 5.6 \dagger$ & $7.3 \pm 4.6 \dagger$ & $8.1 \pm 5.0 \dagger$ & $127.2 \pm 13.6 \dagger$ & $630.6 \pm 92.5 \dagger$ \\
\hline & TM2 & $9.7 \pm 5.8 \dagger$ & $7.7 \pm 4.2 \dagger$ & $8.7 \pm 4.9 \dagger$ & $115.7 \pm 9.2^{*} \S \dagger$ & $624.5 \pm 57.2 \dagger$ \\
\hline & TM3 & $8.6 \pm 5.5 \dagger$ & $6.7 \pm 4.2 \dagger$ & $7.6 \pm 4.8 \dagger$ & $119.3 \pm 7.9+$ & $629.2 \pm 67.8 \dagger$ \\
\hline \multirow[t]{4}{*}{ POST } & NP & $5.1 \pm 3.7 \dagger \ddagger$ & $3.0 \pm 1.9 \dagger \ddagger$ & $4.1 \pm 2.4 † \ddagger$ & $123.3 \pm 12.3 \dagger$ & $520.2 \pm 59.6 \dagger \ddagger$ \\
\hline & TM1 & $3.6 \pm 1.5 \dagger$ & $3.3 \pm 2.1 \dagger$ & $3.5 \pm 1.8 \dagger$ & $123.9 \pm 11.1 \dagger$ & $523.0 \pm 64.0 \dagger \ddagger$ \\
\hline & TM2 & $5.0 \pm 3.9 \dagger \ddagger$ & $2.9 \pm 2.3 † \ddagger$ & $3.9 \pm 2.9 \dagger \ddagger$ & $120.1 \pm 9.6 †$ & $516.7 \pm 54.5 \dagger \ddagger$ \\
\hline & TM3 & $5.5 \pm 3.8 \dagger$ & $3.8 \pm 2.2 \dagger$ & $4.6 \pm 2.8 \dagger$ & $124.0 \pm 12.6 † \ddagger$ & $525.0 \pm 53.5 \dagger \ddagger$ \\
\hline
\end{tabular}

Values given as means \pm SD. Abbreviations: Up, average increasing BRG; Down, average decreasing BRG; Mean, average BRG; SBP, prevailing systolic blood pressure; RRI, prevailing R-R interval; REST, resting; EX, exercise; POST, active recovery; NP, non-pregnant; TM1, $1^{\text {st }}$ trimester; TM2, $2^{\text {nd }}$ trimester; TM3, $3^{\text {rd }}$ trimester. *Significant difference from NP, within condition $(P<$ 0.05). §Significant difference from TM1, within condition $(P<0.05)$. †Significant difference from rest, same group $(P<0.05)$. ‡Significant difference from exercise, same group $(P<0.05)$. 
Table 5. Cardiac time intervals data at rest, during light exercise, and during active recovery following peak exercise throughout pregnancy.

\begin{tabular}{|c|c|c|c|c|c|c|c|c|}
\hline & & $P R$ Int (s) & P Dur (s) & QRS Int (s) & $Q T$ Int (s) & $Q T c(s)$ & JT Int (s) & $\begin{array}{l}\text { Tpeak Tend } \\
\text { Int (s) }\end{array}$ \\
\hline \multirow[t]{4}{*}{ REST } & $N P$ & $166.1 \pm 39.1$ & $102.3 \pm 27.2$ & $68.4 \pm 13.2$ & $367.6 \pm 29.9$ & $396.8 \pm 19.6$ & $298.8 \pm 30.3$ & $59.9 \pm 7.1$ \\
\hline & TM1 & $160.4 \pm 12.0$ & $87.5 \pm 20.2$ & $68.1 \pm 10.0$ & $322.3 \pm 28.7$ & $407.3 \pm 19.3$ & $254.3 \pm 25.7$ & $60.1 \pm 11.5$ \\
\hline & TM2 & $147.4 \pm 28.8$ & $83.3 \pm 26.5$ & $68.2 \pm 14.2$ & $354.5 \pm 19.6$ & $404.5 \pm 19.0$ & $286.4 \pm 24.4$ & $65.2 \pm 11.1$ \\
\hline & TM3 & $171.2 \pm 41.0$ & $101.4 \pm 43.6$ & $66.1 \pm 12.8$ & $335.2 \pm 16.4^{*}$ & $392.8 \pm 15.3$ & $269.2 \pm 17.4^{*}$ & $58.9 \pm 6.5$ \\
\hline \multirow[t]{4}{*}{$E X$} & $N P$ & $167.6 \pm 33.8$ & $99.5 \pm 20.9$ & $72.9 \pm 13.8 \dagger$ & $343.9 \pm 24.6 \dagger$ & $423.8 \pm 21.6 \dagger$ & $270.6 \pm 27.3 \dagger$ & $60.9 \pm 9.7$ \\
\hline & TM1 & $165.5 \pm 8.3$ & $90.2 \pm 12.4$ & $67.2 \pm 8.3$ & $326.0 \pm 28.0 \dagger$ & $411.6 \pm 16.2$ & $259.0 \pm 32.7 \dagger$ & $60.0 \pm 10.0$ \\
\hline & TM2 & $143.5 \pm 29.8$ & $79.7 \pm 27.5$ & $73.4 \pm 14.1$ & $334.0 \pm 22.2 \dagger$ & $422.7 \pm 18.6$ & $260.6 \pm 26.1 \dagger$ & $65.6 \pm 12.0$ \\
\hline & TM3 & $168.8 \pm 63.9$ & $89.2 \pm 15.5$ & $70.5 \pm 14.8$ & $324.7 \pm 14.8$ & $410.2 \pm 15.2$ & $254.2 \pm 17.1 \dagger$ & $59.7 \pm 8.8$ \\
\hline \multirow[t]{4}{*}{ POST } & $N P$ & $152.1 \pm 28.9$ & $89.4 \pm 24.8$ & $76.4 \pm 13.1 \dagger \ddagger$ & $303.8 \pm 38.1 † \ddagger$ & $434.6 \pm 62.7 \dagger$ & $227.4 \pm 40.7 \dagger \ddagger$ & $50.6 \pm 10.4 \dagger \neq$ \\
\hline & TM1 & $150.2 \pm 32.8$ & $78.4 \pm 31.0$ & $70.5 \pm 8.2 \dagger$ & $291.3 \pm 21.3 † \ddagger$ & $408.9 \pm 17.9$ & $220.8 \pm 25.3 † \ddagger$ & $55.7 \pm 11.3 \dagger$ \\
\hline & TM2 & $138.1 \pm 27.6$ & $74.1 \pm 21.3$ & $72.9 \pm 14.2 \dagger$ & $302.1 \pm 22.3 † \ddagger$ & $422.3 \pm 32.9$ & $228.7 \pm 23.9 \dagger \ddagger$ & $56.8 \pm 8.9 \dagger \ddagger$ \\
\hline & TM3 & $145.0 \pm 24.9$ & $84.1 \pm 19.7$ & $71.4 \pm 13.6 \dagger$ & $304.9 \pm 27.6 \dagger \ddagger$ & $425.5 \pm 49.5 \dagger$ & $233.2 \pm 30.1 \dagger \ddagger$ & $56.6 \pm 9.3$ \\
\hline
\end{tabular}

Values given as means \pm SD. Abbreviations: PR Int, P-R interval; P Dur, duration of p wave; QRS Int, QRS interval; QT Int, Q-T interval; QTc, rate-corrected QT interval; JT Int, J-T interval; Tpeak Tend Int, duration from T wave peak to end; REST, resting; EX, exercising; POST, recovery; NP, non-pregnant; TM1, $1^{\text {st }}$ trimester; TM2, $2^{\text {nd }}$ trimester; TM3, $3^{\text {rd }}$ trimester. *Significant difference from NP, within condition $(P<0.05) \dagger$ Significant difference from rest, same group $(P<0.05)$. $\ddagger$ Significant difference from exercise, same group $(P<0.05)$. 


\section{Figure Captions}

14 Figure 1. Change in heart rate variability, quantified by the root mean squared of successive

15 differences (RMSSD), between rest, steady-state light exercise (EX), and active recovery

16 following peak exercise (POST) throughout pregnancy. Abbreviations: NP, non-pregnant; TM1,

$17 \quad 1^{\text {st }}$ trimester; TM2, $2^{\text {nd }}$ trimester; TM3, $3^{\text {rd }}$ trimester. $*$ denotes significant difference compared to

18 corresponding NP change in $\operatorname{RMSSD}(p<0.05)$.

19 Figure 2. Change in mean spontaneous cardiovagal baroreflex gain (BRG) between rest, steady-

20 state light exercise (EX), and active recovery following peak exercise (POST) throughout

21 pregnancy. Abbreviations: NP, non-pregnant; TM1, $1^{\text {st }}$ trimester; TM2, $2^{\text {nd }}$ trimester; TM3, $3^{\text {rd }}$

22 trimester. * denotes significant difference compared to corresponding NP change in baroreflex

23 gain $(p<0.05)$. 


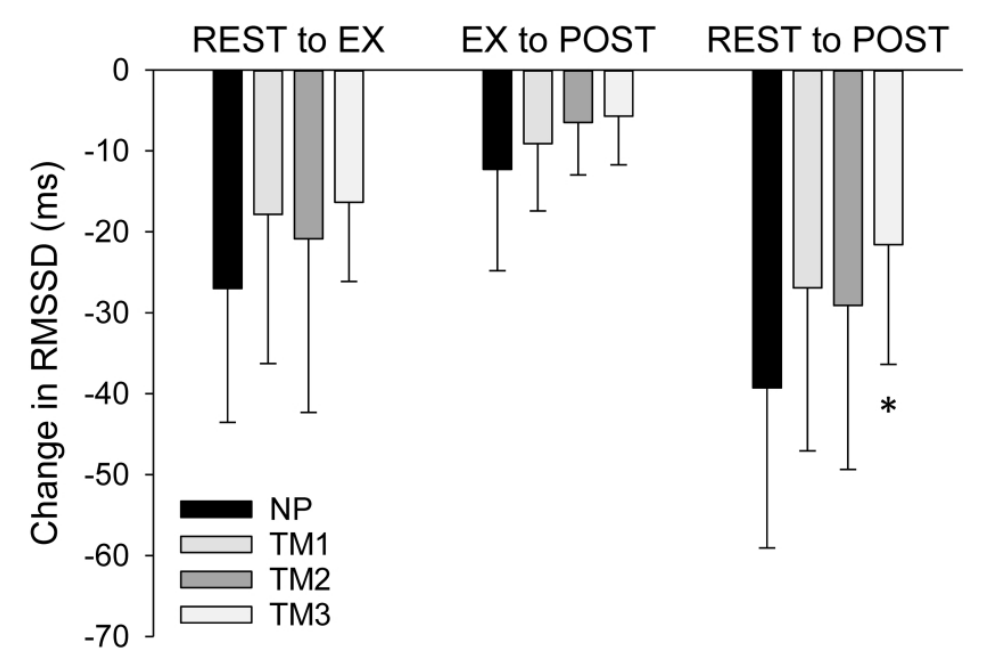

Figure 1. Change in heart rate variability, quantified by the root mean squared of successive differences (RMSSD), between rest, steady-state light exercise (EX), and active recovery following peak exercise (POST) throughout pregnancy.

$338 \times 190 \mathrm{~mm}(300 \times 300 \mathrm{DPI})$ 


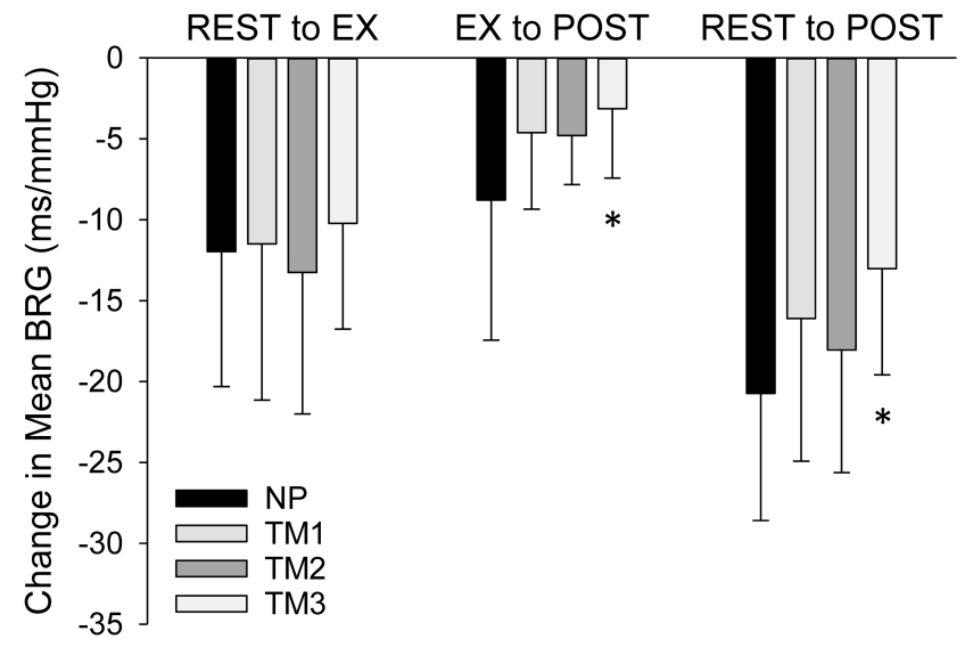

Figure 2. Change in mean spontaneous cardiovagal baroreflex gain (BRG) between rest, steady-state light exercise (EX), and active recovery following peak exercise (POST) throughout pregnancy.

$338 \times 190 \mathrm{~mm}(300 \times 300 \mathrm{DPI})$ 